\title{
Characteristics of Cortical Activation in Schizophrenia during the Card Game "Concentration"
}

\author{
RYO FUJIKI****, KIICHIRO MORITA*,**, MASAYUKI INOUE**, YOSHIHISA SHOJI*,**, \\ KEIICHIRO MORI***, YOHEI ISHII**, CHIZUKO KAWABE**, \\ ATSUSHI YAMAMOTO**, AND NAOHISA UCHIMURA* \\ *Department of Neuropsychiatry and **Cognitive and Molecular Research Institute of Brain Diseases, \\ Kurume University School of Medicine, Kurume 830-0011, Japan
}

Received 22 November 2012, accepted 27 December 2012

Edited by TAKAYUKI TANIWAKI

\begin{abstract}
Summary: Various functional imaging studies have demonstrated reduced lateralization of cortical activation during neurocognitive tasks in schizophrenia. -A well-known card game, "concentration", reflects working memory (WM). We compared characteristics of hemodynamic changes in the prefrontal to temporo-parietal areas of the brain during this card game between 24 outpatients with schizophrenia and 24 age- and gendermatched healthy control subjects. Using multi-channel near-infrared spectroscopy, we measured relative changes in oxygenated hemoglobin concentration (oxy-Hb changes), which reflects brain activity during this task. Patients showed reduced lateralization in the midfrontal area, which is involved in executive functions, and in the inferior parietal area, involved in WM subcomponents. We also found a significant negative correlation between left midfrontal region oxy-Hb changes and severity of negative symptoms of schizophrenia. Our results characterized disease-related features, suggesting the usefulness of oxy-Hb change measurement during this card game for assessing functional outcome in schizophrenic patients.
\end{abstract}

Key words card game "concentration", multi-channel near-infrared spectroscopy, reduced lateralization, schizophrenia, working memory

\section{INTRODUCTION}

The card game "concentration" (also known as "matching pairs" or "pelmanism") involves turning over pairs of cards with the aim of locating cards with matching numbers. This requires players to retain information about cards and manipulate the stored information. Jimison et al. [1] used this card game as a tool for measuring working memory (WM). WM is a limited-capacity system involved in temporary storage and manipulation of information [2]. This activity is a high-order cognitive function that is essential to daily life. Much is known about WM deficits in patients with schizophrenia, which have been shown to persist even when clinical symptoms decrease, and they are suggested to be important for their clinical outcome [3-6]. Previous studies have demonstrated reduced lateralization of activation during WM tasks [7-9] and correlation between degree of WM impairment and negative symptoms in schizophrenic patients $[4,10]$.

Tupak et al. [11] revealed lateralization of activation during a semantic verbal fluency task in healthy subjects. Lee et al. [7] demonstrated reduced lateralization during a spatial WM task in patients with schizophrenia, suggesting that this finding might be the re-

Address correspondence to: Ryo Fujiki, M.D. Department of Neuropsychiatry and Cognitive and Molecular Research Institute of Brain Diseases, Kurume University School of Medicine, 67 Asahi-Machi, Kurume-City, Japan. Tel: 81-942-31-7564 Fax: 81-942-35-6041 E-mail: fujiki_ryou@med.kurume-u.ac.jp 
sult of a compensatory mechanism for WMdysfunction. As this dysfunction is shown throughout the various stages of illness, it is important to detect it early. In addition to increased activation of the right parietal cortex during visuospatial maintenance in schizophrenia, Henseler et al. [12] (2009) reported that more severely affected patients had stronger prefrontal activation and poorer performance.

We have explored neuroimaging methods using an easy and inexpensive visuospatial WM task to investigate characteristics of schizophrenia. In this study, we compared hemodynamic changes between healthy subjects and patients while actually playing cards on a table. The game is well known and has simple rules. Therefore, it may be an appropriate neurocognitive model for measuring cortical activity enhancement during WM tasks encountered in daily life.

Near-infrared spectroscopy (NIRS) is a practical, noninvasive imaging method employing scattered near-infrared light to measure changes in oxygenated blood hemoglobin at the surface of the brain. The underlying principle is that relative changes in oxygenated hemoglobin concentration (oxy-Hb changes) reflect regional cerebral blood volume, which is associated with brain activity. NIRS has been increasingly used to investigate schizophrenia because the apparatus is relatively small and portable, is easily set up, and imposes minimal limitation of movement [1318]. However, most NIRS studies during WM tasks have focused on frontal areas [14-18]; specific findings in other cortical areas have been unclear.

In this study, we measured oxy-Hb changes during the card game "concentration" using multi-channel
NIRS covering the prefrontal to temporo-parietal areas to investigate characteristics of schizophrenia. The primary aim of the present study was to determine using NIRS whether the card game "concentration" was useful as a neurocognitive task and to identify dissociated activity patterns in patients with schizophrenia compared with those in healthy subjects during this task. We hypothesized that there would be reduced lateralization of activity in patients, as in previous studies. An additional aim was to investigate the relationships between functional brain activity and psychiatric symptoms.

\section{METHODS}

\section{Participants}

Study patients included 24 Japanese outpatients with schizophrenia (15 male and 9 female; mean age, $30.0 \pm 6.0$ years) and 24 age- and gender-matched healthy control subjects (15 male and 9 female; mean age, 29.0 4.4 years). Two attending psychiatrists diagnosed patients using the International Classification of Disease, tenth revision (ICD-10) [19]. All subjects were right-handed according to the Edinburgh Inventory [20]. Their mean intelligence quotient (IQ) values were evaluated by the Japanese version of the National Adult Reading Test (JART) [21]. No subject had any history of neurologic disorder, substance abuse, alcohol abuse, seizure, or head injury. Healthy control subjects had no history of psychiatric illness or familial history of psychosis. All patients were taking atypical antipsychotic medications (i.e. olanzapine $(n=8)$, risperidone $(n=7)$, aripiprazole $(n=3)$, quetiap-

TABLE 1.

Subject characteristics

\begin{tabular}{lccc}
\hline & Patients & Controls & p value \\
\hline Age (year) & $30.0 \pm 6.0$ & $29.0 \pm 4.4$ & 0.48 \\
Gender (F/M) & $9 / 15$ & $9 / 15$ & \\
Education (year) & $13.3 \pm 15$ & $16.5 \pm 1.2$ & $<0.01^{*}$ \\
$\quad$ IQ & $100.3 \pm 10.1$ & $106.2 \pm 6.1$ & 0.07 \\
Illness Duration (year) & $5.1 \pm 2.6$ & & \\
PANSS score & & & \\
$\quad$ Positive subscale & $20.7 \pm 2.9$ & & \\
$\quad$ Negative subscale & $19.7 \pm 3.6$ & & \\
$\quad$ Total & $45.0 \pm 4.4$ & & \\
Medication & & & \\
$\quad$ HPD equivalent (mg/day) & $6.0 \pm 2.8$ & & \\
\hline
\end{tabular}

Data are presented as means \pm SD. $p<0.05$, comparing patients with controls. Abbreviation: HPD, haloperidol. 
ine $(n=2)$, perospirone $(n=2)$, blonanserin $(n=2)$. The mean daily antipsychotic dose in terms of haloperidol (HPD) equivalent was $6.0 \pm 2.8 \mathrm{mg}$ [22]. Psychopathology was assessed using the Positive and Negative Syndrome Scale (PANSS) [23]. Neither patients nor control subjects showed visual disabilities. The ethics committee of Kurume University approved the present study. Written informed consent was obtained from all subjects prior to participation in the study. Demographic and clinical characteristics of all subjects are shown in Table 1.

\section{Measures}

\section{NIRS measurement}

Oxy-Hb changes, which were calculated from the difference in absorbance based on the modified BeerLambert law, were measured during tasks using a multi-channel NIRS machine (ETG4000; Hitachi, Tokyo) from 22 left- and 22 right-sided channels (total, 44) [24]. Distances between the injector and the detector probe were $3 \mathrm{~cm}$. We defined each measurement area between probes as a channel (ch). The NIRS machine is thought to measure changes at points 2 to 3 $\mathrm{cm}$ beneath the scalp, that is, the cerebral cortical surface $[25,26]$. The lowest anterior probes, right ch 22

a
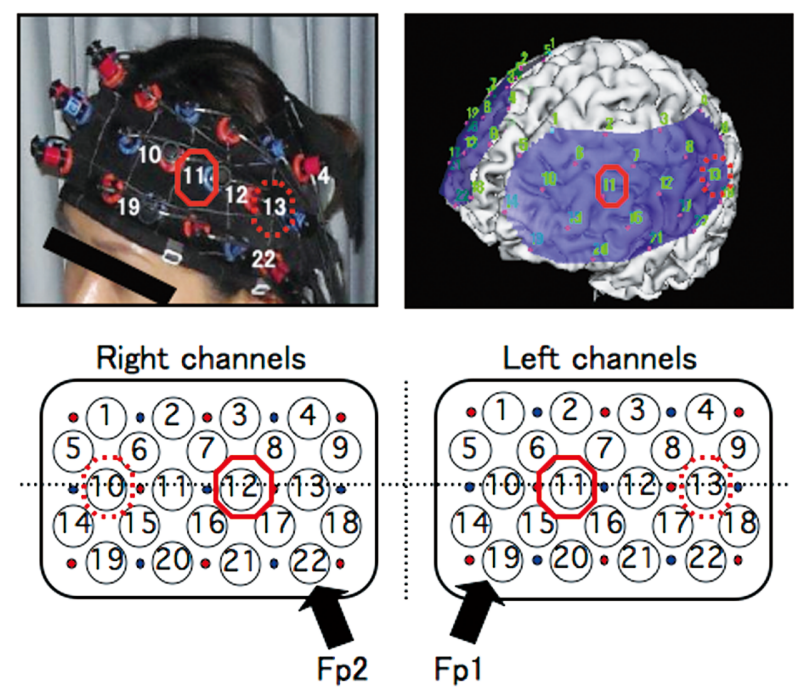

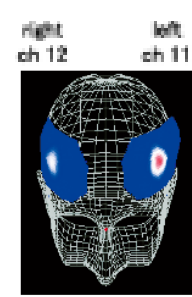

controle

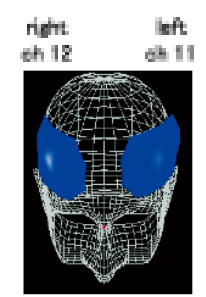

satients

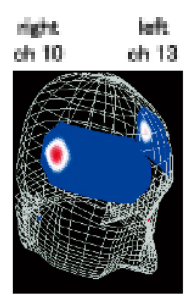

controle

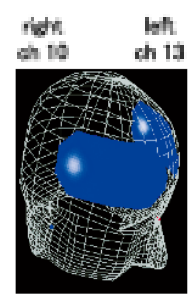

setients and left ch 19, were positioned along the Fp1-Fp2 line according to the International 10-20 system used in electroencephalography (Fig. 1a). In the present study, all subjects were instructed to minimize their movements and jaw fixation was used during the task to avoid movement artifacts. NIRS data were calculated from the mean waves of oxy-Hb changes at each recording site and region of interests (ROIs), which corresponded to presumed sites of WM function. ROIs evaluated to determine physiologic significance were selected for channels reflecting the midfrontal area and inferior parietal area. The midfrontal area has been reported to reflect executive function, and the inferior parietal area to reflect verbal and visuospatial WM [27-29]. The pre-task baseline was determined as the mean during the $10 \mathrm{sec}$ preceding the task period, while the post-task baseline was determined as the mean during the 30-40 sec after the task period. Linear fitting was applied to the data between these two baselines.

\section{Right-hand motion task}

As an initial study, we investigated a right-hand motion task to confirm the relationship between activation region and anatomic region. Twenty-four right-

b

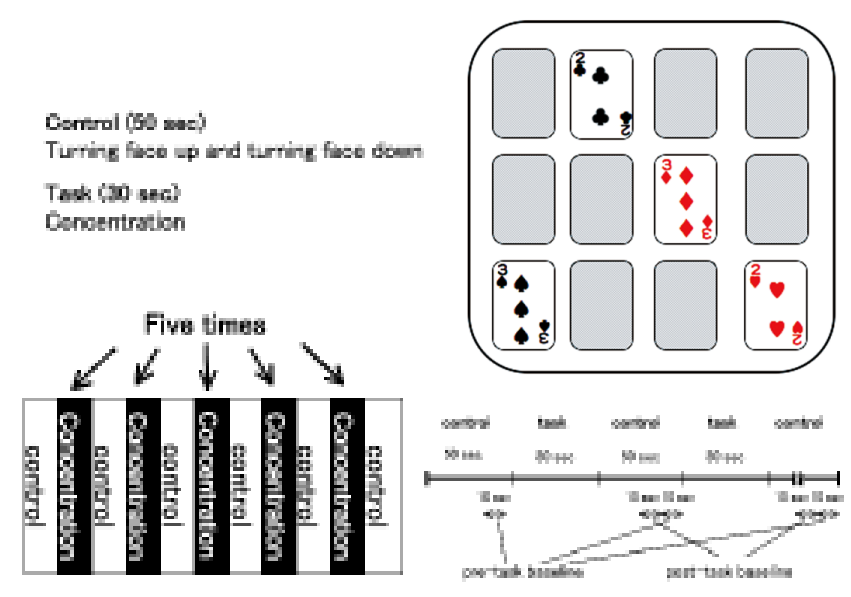

Fig. 1. Study design: a, Location of channels: b, protocol Each subject performed one session, consisting of five blocks and tried to make six pairs during the $30 \mathrm{sec}$ task period. They played the "concentration" game using cards actually on a table. When the control period was finished, we put out 12 cards ( 6 pairs) arranged face down at random, and when task period was finished, we put out 12 identical cards (aces). There was a start signal when the new 12 cards were put out. 
handed healthy control subjects participated in the activation task. In the right-hand motion task, 12 cards bearing the same number (ace) were arranged on the table, and subjects were instructed to repeat turning them face up and turning them face down for 2 cards in order, using only the right hand. As a control condition, all subjects were asked to relax with their eyes open and their hands still. Averaged waveforms for 5 of these blocks were measured as one session, and oxy-Hb changes from the control condition were expressed numerically for each 100 milliseconds, and the area of the task period $(30 \mathrm{sec})$ was calculated as data.

\section{Activation task}

Brain activation was measured during the card game "concentration". For the activation task, each subject sat on a comfortable chair and had to perform the task using actual playing cards on a table. They were instructed to use only the right hand. One session consisted of 2 contrasting conditions (concentration task, control condition), and all subjects alternated between these conditions. The concentration task was performed as 5 blocks in 1 session. In the concentration task, subjects searched for identically numbered card pairs among 12 cards placed face down in front of them. Subjects were instructed to select matching numbers irrespective of color, suit, or other markings. In the control condition, 12 cards bearing the same number (ace) were used, and subjects were instructed to repeat turning them face up and turning them face down for 2 cards in order. According to different time windows for control and task conditions, the control period took more than $30 \mathrm{sec}$ to recovery of activation. Therefore, we set the control period at $50 \mathrm{sec}$ in order to ensure sufficient recovery time. Averaged waveforms for 5 blocks were analyzed as 1 session using the "Integral mode" of the machine. Oxy-Hb changes between activation and control periods were expressed numerically for each 100 milliseconds. The area of the task period (30 sec) was calculated (Fig. 1b). We used values from this area as our NIRS data. The total number of cards paired was used as the performance for a session.

\section{Relationship between recording site and anatomic region}

We investigated the right-hand motion task during NIRS to confirm each channel of ROI. NIRS data were converted to a normalized brain image template (3 dimensional composition indication unit: Hitachi, Tokyo). In the right-hand motion task, oxy-Hb change at all left recording sites was $13.4 \pm 24.3 \mathrm{mmol}^{*} \mathrm{~mm}$ and that at all right recording sites was $8.2 \pm 25.3$ mmol*mm. Significant differences were obtained between the left ch 3 and the right ch $2(\mathrm{~F}=7.62, \mathrm{p}<0.01)$, and between the left ch 8 and the right ch $6(\mathrm{~F}=4.77$, $\mathrm{p}<0.04)$. Oxy-Hb changes at each of these left channels (left ch 3, left ch 8) were significantly larger than those at the corresponding right channel (right ch 2, right ch 6 , respectively). Since these left channels were thought to be associated with neural activity in the left motor cortex due to right-hand motion, ROIs reflecting the midfrontal area and inferior parietal area were speculated to be left ch 11 and right ch 12, and left ch 13 and right ch 10 , respectively.

\section{Statistical analysis}

Mean data from each group were used in the analysis (healthy subjects; $n=24$, patients; $n=24$ ). NIRS data were examined using three-way analysis of variance (ANOVA) (group $\times$ hemisphere $\times$ recording sites) to evaluate the group main effects. Whether interaction was obtained or not, two-way ANOVA (group $X$ hemisphere) was evaluated in all recording sites and ROIs. Finally, whether interaction was obtained or not, one-way ANOVA (group) was evaluated by hemisphere or for all recording sites and ROIs. Furthermore, one-way ANOVA (hemisphere) was evaluated in groups or all recording sites and ROIs. Fisher's protected least significant difference (PLSD) was then applied as a post hoc test between sites or groups. The correlation between the oxy-Hb changes and the PANSS score is expressed as Pearson's product-moment correlation coefficient; Bartlett's test was used to evaluate statistical significance. A value of $\mathrm{p}<0.05$ was accepted as statistically significant. Values are expressed as the mean \pm standard deviation (SD). We used the oxy-Hb changes as data in this study because these changes correlate more strongly with BOLD of functional magnetic resonance imaging (fMRI) than do deoxy-Hb changes [30].

\section{RESULTS \\ Performance on "concentration" game task}

Task performance was $18.8 \pm 4.3$ in patients and $21.9 \pm 6.6$ in controls, showing no significant difference between groups $(\mathrm{F}=3.65, \mathrm{p}>0.06)$ (Table 2$)$.

\section{"Concentration" game task}

$\mathrm{Oxy}-\mathrm{Hb}$ change at all left recording sites was $9.30 \pm 20.2 \mathrm{mmol}^{*} \mathrm{~mm}$ and that at all right recording sites was $10.0 \pm 22.8 \mathrm{mmol}^{*} \mathrm{~mm}$ during the game task in patients, compared with $23.5 \pm 35.3 \mathrm{mmol} * \mathrm{~mm}$ and 
$25.0 \pm 33.1 \mathrm{mmol}^{*} \mathrm{~mm}$ in controls, respectively. In all recording sites, three-way ANOVA (group $\times$ hemisphere $\times$ recording sites) revealed a significant main effect of 'group' $(\mathrm{F}=147, \mathrm{p}<0.01)$, but no significant interactions. Two-way ANOVA (group $\times$ hemisphere) revealed a significant main effect of 'group' $(\mathrm{F}=137$, $\mathrm{p}<0.01$ ), but no significant interactions. One-way ANOVA revealed significantly smaller values for patients than for controls on both sides (left, $\mathrm{F}=64.1$, $\mathrm{p}<0.01$; right: $\mathrm{F}=72.6, \mathrm{p}<0.01)$. No significant lateralization of oxy- $\mathrm{Hb}$ changes at any recording site was observed in either group (controls, $\mathrm{p}=0.50$; patients, $\mathrm{p}=0.60$ ).

In ROI, three-way ANOVA (group $\times$ hemisphere $\times$ recording sites) revealed a significant main effect of 'group' $(\mathrm{F}=29.8, \mathrm{p}<0.01)$ as well as significant interactions of 'group $\times$ hemisphere $\times$ recording sites' $(\mathrm{F}=9.80, \mathrm{p}<0.02)$. Oxy-Hb change at left ch 11 was $6.90 \pm 14.7 \mathrm{mmol}^{*} \mathrm{~mm}$ and that at right $\mathrm{ch} 12$ was

TABLE 2.

Comparison between "concentration" task results in patients with schizophrenia and healthy subjects

\begin{tabular}{|c|c|c|c|c|}
\hline & \multicolumn{2}{|c|}{ Patients $(n=24)$} & \multicolumn{2}{|c|}{ Controls $(n=24)$} \\
\hline & Mean & SD & Mean & SD \\
\hline Performance & 18.8 & 4.3 & 21.9 & 6.6 \\
\hline \multicolumn{5}{|l|}{$\begin{array}{l}\text { Oxy-Hb change } \\
{\left[\mathrm{mmol}^{*} \mathrm{~mm}\right]}\end{array}$} \\
\hline Left ch 11 & 6.9 & 14.7 & 36.7 & 27.9 \\
\hline Right ch 12 & 12.4 & 21.5 & 21.1 & 16.6 \\
\hline Left ch 13 & 12.6 & 23.0 & 21.4 & 31.8 \\
\hline Right ch 10 & 12.3 & 23.6 & 41.2 & 29.6 \\
\hline
\end{tabular}

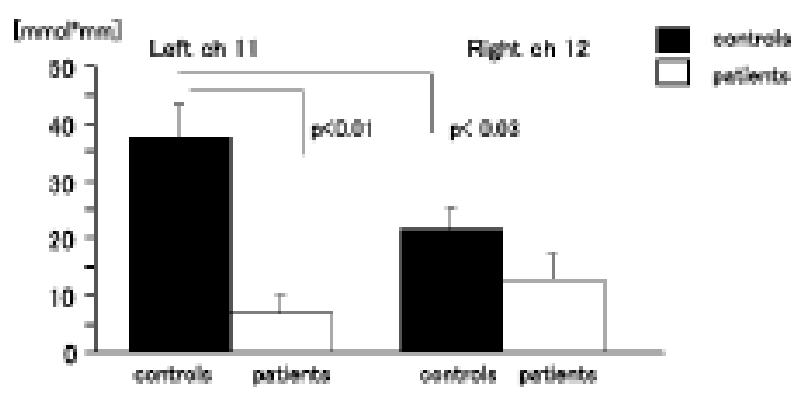

Fig. 2. Oxy-Hb changes appear along the ordinate and groups along the abscissa. Oxy-Hb changes at left ch 11 in patients $(\square)$ were significantly smaller than in healthy controls (ם). Oxy-Hb changes at left ch 11 were significantly larger than those at right ch 12 in controls, but not in patients.
$12.4 \pm 21.5 \mathrm{mmol} * \mathrm{~mm}$ in patients. In controls, oxy- $\mathrm{Hb}$ change at left ch 11 was $36.7 \pm 27.9 \mathrm{mmol} * \mathrm{~mm}$; at right ch 12 , this was $21.1 \pm 16.6 \mathrm{mmol}^{*} \mathrm{~mm}$ (Table 2). In left ch 11 and right ch 12, two-way ANOVA (group $\times$ hemisphere) revealed a significant main effect of 'group' $(\mathrm{F}=20.5, \mathrm{p}<0.01)$ as well as a significant interaction of 'group $\times$ hemisphere' $(\mathrm{F}=6.16, \mathrm{p}=0.01)$. One-way ANOVA revealed that oxy-Hb changes at left ch 11 were significantly larger than those at right ch 12 in controls $(\mathrm{F}=5.52, \mathrm{p}=0.02)$, but no significant difference was found in patients $(\mathrm{F}=1.07, \mathrm{p}=0.31)$. In addition, oxy-Hb changes at left ch 11 in controls were significantly larger than in patients $(\mathrm{F}=21.4, \mathrm{p}<0.01)$, but no significant difference was found between groups at right ch $12(\mathrm{~F}=2.45, \mathrm{p}=0.12)$ (Fig. 2).

Oxy-Hb change in patients at left ch 13 was $12.6 \pm$ $23.0 \mathrm{mmol}^{*} \mathrm{~mm}$; at right $\mathrm{ch} 10$, this was $12.3 \pm 23.6$ mmol*mm. In controls, oxy-Hb change at left ch 13 was $21.4 \pm 31.8 \mathrm{mmol}^{*} \mathrm{~mm}$, while at right $\mathrm{ch} 10$, this was $41.2 \pm 29.6 \mathrm{mmol}^{*} \mathrm{~mm}$ (Table 2). In left ch 13 and right ch 10, two-way ANOVA (group $\times$ hemisphere) revealed a significant main effect of 'group' ( $\mathrm{F}=11.1$, $\mathrm{p}<0.01)$ as well as a significant interaction of 'group $\times$ hemisphere' $(\mathrm{F}=3.99, \mathrm{p}<0.05)$. One-way ANOVA revealed that oxy-Hb changes at left ch 13 were significantly smaller than those at right ch 10 in controls $(\mathrm{F}=5.02, \mathrm{p}=0.03$ ), but no significant difference was found in patients $(\mathrm{F}=0.001, \mathrm{p}=0.97)$. In addition, oxy$\mathrm{Hb}$ changes at right ch 10 in controls were significantly larger than in patients $(\mathrm{F}=14.0, \mathrm{p}<0.01)$, but no significant difference between groups was found at left ch $13(\mathrm{~F}=1.20, \mathrm{p}=0.28)$ (Fig. 3).

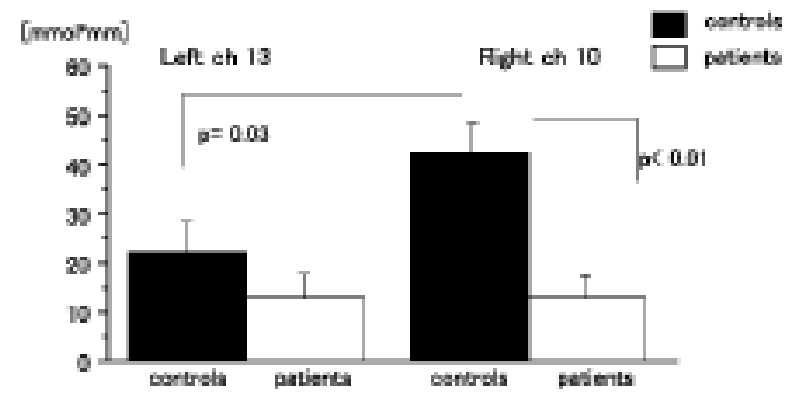

Fig. 3. Oxy-Hb changes appear along the ordinate and groups along the abscissa. Oxy-Hb changes at right ch 10 in patients $(\square)$ were significantly smaller than in healthy controls $(\square)$. Oxy-Hb changes at right ch 10 were significantly larger than those at left ch 13 in controls, but not in patients. 


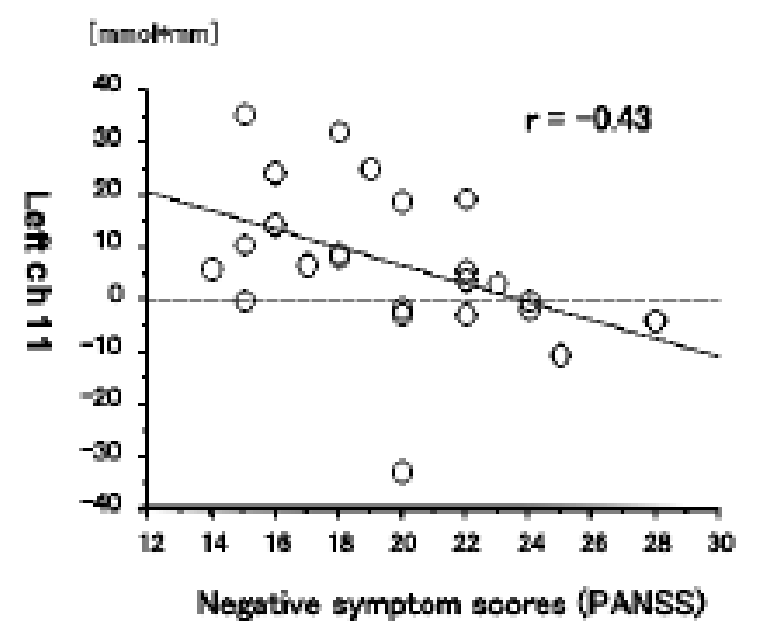

Fig. 4. Relationship between oxy-Hb changes and negative symptom scores.

Oxy-Hb changes at left ch 11 showed a significant negative correlation with negative symptom scores in patients. The correlation coefficient $(\mathrm{r})$ was $-0.43(\mathrm{p}=$ 0.03 ) at left ch 11.

\section{Relationship between oxy-Hb changes and PANSS scores}

Oxy-Hb changes at left ch 11 showed a significant negative correlation with negative symptom scores in patients. The correlation coefficient was $-0.43(\mathrm{p}=0.03)$ at left ch 11 (Fig. 4). On the other hand, oxy-Hb changes did not correlate with positive symptom scores, total symptom scores, or antipsychotic drug dose. Moreover, oxy-Hb changes were not correlated with performance in either group.

\section{DISCUSSION}

This study compared regional oxy-Hb changes between patients with schizophrenia and healthy control subjects during a task involving the card game "concentration". This task requires attention and executive control, and it is considered to involve WM. Recent neuroimaging studies during neurocognitive tasks have examined anatomic regions related to WM functions, suggesting that the phonologic loop involved in WM is associated mainly with the left inferior parietal region \{Brodmann's area (BA) 40\} and Broca's area (BA 6/44) [27,31,32], while the visuospatial sketchpad in WM is associated mainly with the right inferior parietal region (BA 40), premotor cortex (BA 6), inferior frontal cortex (BA 47), and visual association area (BA 19) $[27,31,32]$. The central executive system is considered to involve mainly left and right dorsolateral prefrontal areas (BA 9/46) [33]. Thus, the mid- frontal area has been thought to reflect executive function, and the inferior parietal area to reflect both verbal and visuospatial WM in common.

In the present study, we first investigated the relationship between anatomic region and channel, using a right-hand motion task. The left motor cortex was associated with right-hand motion, specifically around ch 3 and ch 8 , corresponding to BA 4. Therefore, left ch 11 and right ch 12 corresponded to the midfrontal area including BA 9/46. Left ch 13 and right ch 10 represented the inferior parietal area, including BA 40. In previous studies, reduced lateralization of activation during WM tasks was reported in patients with schizophrenia [6-8]. This finding suggests reduced WM in patients [9]. Nakahachi et al. [14] (2010) evaluated working memory using the advanced trail-making test (ATMT) in healthy subjects, and observed bilateral activities during ATMT task F [14]. In the present study, reduced lateralization at all recording sites was observed in both groups. This finding is thought to reflect use of not only visual but also verbal information in the task, as also occurs during ATMT task F. The various other features of the card gave clues for memorizing the card's position, which may have reflected functions including verbal working memory.

Regarding the ROI in the area assumed to be the midfrontal region (left ch 11, right ch 12), oxy-Hb changes at the left recording site were significantly greater in controls than in patients. Oxy-Hb changes at left ch 11 were significantly greater than those at right ch 12 in controls, while no laterality was observed in patients, which may have reflected schizophrenia-associated reduction of executive function. In the area taken to be the inferior parietal region (left ch 13 and right ch 10), oxy-Hb changes at the right recording site were significantly greater in controls than in patients. Oxy-Hb changes at right ch 10 were significantly greater than those at left ch 13 in controls, while no laterality was observed in patients. The inferior parietal region is related to verbal and visual working memory [27,31]. However, in the present task, we suspected that healthy subjects showed right hemisphere predominance because visuospatial information retention was more important than verbal information, while patients showed reduced lateralization because of visuospatial and verbal WM deficits in comparison with controls. Our examination showed reduced lateralization of activation at ROIs, while performance did not differ significantly between groups. This finding may reflect WM deficits and possibly also compensation for cortical dysfunction, as suggested in a previous report [7]. 
In previous studies, a correlation was observed between WM in patients and the negative symptom assessment score $[4,10]$. Meanwhile, WM deficits have been proposed as a functional marker of schizophrenia $[34,35]$. In our study, although there was no correlation between performance and any subcomponent of PANSS score, a negative correlation was observed between negative symptom assessment score and oxy$\mathrm{Hb}$ changes at left ch 11 . That is, patients with more severe negative symptoms have weaker activation of the area reflecting executive function. Our finding suggests that deficient local blood flow changes at left ch 11 may serve as a biologic marker of schizophrenia. Although further studies are necessary, this marker might serve as a simple, objective evaluation tool, providing a useful way to predict social functions, and to decide when to introduce rehabilitation. However, our study had several limitations: 1) Education years were not controlled. Although our data showed no significant difference between education and performance or activation, we believe that future studies should take this issue into account. 2) Anatomic region. It is assumed that the spatial resolution of the NIRS is poor. We performed a right-hand motion task and speculated on the relationship between anatomic region and channel from activation of right-hand movement. However, our NIRS data were not analyzed in terms of individual MRI brain scans. 3) Body movement. NIRS does not restrict body movement and can be applied in a natural posture, but artifacts are likely to be produced by nodding and rotation of the head. We therefore employed jaw fixation and data averaging to reduce artifacts as much as possible. 4) Influences of medications. All patients received atypical anti-psychotic drugs, with which WM deficits have been associated [36,37]. However, WM deficits have been reported in untreated patients with schizophrenia, in patients about to begin treatment, and in patients at clinical onset of schizophrenia, as well as in healthy first-degree relatives of patients with schizophrenia $[4,35,38,39]$. The relationship between medication effects and WM is unclear. Future studies should investigate drug-naive patients.

\section{CONCLUSION}

To our knowledge, this is the first study to perform NIRS during tasks associated with the card game "concentration". This study showed reduced lateralization of activation in the midfrontal area and the inferior parietal area in patients with schizophrenia. We suggest that these findings may be disease-related features.
Thus, this actual playing card game is useful as a neurocognitive task. In addition, an observed relationship between oxy-Hb changes and negative symptom scores may be of help in measuring and predicting functional outcome in patients.

ACKNOWLEDGMENTS: This research was supported in part by a grant (20591425) from the Japanese Grant-in-Aid Fund for Science Research.

\section{REFERENCES}

1. Jimison HB, MacKanna J, Ambert K, Hagler S, Hatt WJ et al. Models of cognitive performance based on home monitoring data. Conf Proc IEEE Eng Med Biol Soc 2010; 5234-5237.

2. Baddeley AD, and Hitch, GJ. Working memory, in Bower GA. (Ed.), Recent Advances in Learning and Motivation. Academic Press New York: pp 47-90 1974.

3. Park S, Püschel J, Sauter B, Rentsch M, and Hell D. Spatial working memory deficits and clinical symptoms in schizophrenia patients; A 4-months follow-up study. Biol Psychiatry 1999; 46:392-400.

4. Park S, Püschel J, Sauter B, Rentsch M, and Hell D. Visual object working memory function and clinical symptoms in schizophrenia. Schizophr Res 2002; 59:261-268.

5. Silver H, Feldman P, Bilker W, and WGur RC. Working memory deficit as a core neuropsychological dysfunction in schizophrenia. Am J Psychiatry 2003; 160:1809-1816.

6. Zilles D, Gruber E, Falkai P, and Gruber O. Patients with schizophrenia show deficits of working memory maintenance components in circuit-specific tasks. Eur Arch Psychiatry Clin Neurosci 2010; 260:519-525.

7. Lee J, Folley BS, Gore J, and Park S. Origins of spatial working memory deficits in schizophrenia: An eventrelated fMRI and near-infrared spectroscopy study. PLoS One 2008; 3:e1760.

8. Menon V, Anagnoson RT, Mathalon DH, Glover GH, and Pfefferbaum A. Functional neuroanatomy of auditory working memory in schizophrenia: relation to positive and negative symptoms. Neuroimage 2001; 13:433-446.

9. Walter H, Wunderlich AP, Blankenhorn M, Schäfer S, Tomczak R et al. No hypofrontality, but absence of prefrontal lateralization comparing verbal and spatial working memory in schizophrenia. Schizophr Res 2003; 61:175184.

10. Carter C, Robertson L, Nordahl T, Chaderjian M, Kraft L et al. Spatial working memory deficits and their relationship to negative symptoms in unmedicated schizophrenia patients. Biol Psychiatry 1996; 40:930-932.

11. Tupak SV, Badewien M, Dresler T, Hahn T, Ernst LH et al. Differential prefrontal and frontotemporal oxygenation patterns during phonemic and semantic verbal fluency. Neuropsychologia 2012; 50(7):1565-1569.

12. Henseler I, Falkai P, and Gruber O. A systematic fMRI investigation of the brain systems subserving different working memory components in schizophrenia. Eur J Neurosci 2009; 30:693-702.

13. Ehlis AC, Herrmann MJ, Plichta MM, and Fallgatter AJ. 
Cortical activation during two verbal fluency tasks in schizophrenic patients and healthy controls as assessed by multi-channel near-infrared spectroscopy. Psychiatry Re Neuroimaging 2007; 156:1-13.

14. Nakahachi T, Ishii R, Iwase M, Cznuet L, Takahashi H et al. Frontal cortex activation associated with speeded processing of visuospatial working memory revealed by multichannel near-infrared spectroscopy during Advanced Trail Making Test performance. Behav Brain Res 2010; 215:2127.

15. Shibuya-Tayoshi S, Sumitani S, Kikuchi K, Tanaka T, Tayoshi $\mathrm{S}$ et al. Activation of the prefrontal cortex during the Trail-Making Test detected with multichannel near-infrared spectroscopy. Psychiatry Clin Neurosci 2007; 61:616621.

16. Sumitani S, Tanaka T, Tayoshi T, Ota K, Kameoka N et al. Hemodynamic changes in the prefrontal cortex during mental works as measured by multi channel near-infrared spectroscopy (NIRS). J Med Invest 2005; 52 Suppl:302-303.

17. Suto T, Fukuda M, Ito M, Uehara T, and Mikuni M. Multichannel near-infrared spectroscopy in depression and schizophrenia: cognitive brain activation study. Biol Psychiatry 2004; 55:501-511.

18. Watanabe A, and Kato T. Cerebrovascular response to cognitive tasks in patients with schizophrenia measured by nearinfrared spectroscopy. Schizophr Bull 2004; 30:435-444.

19. World Health Organization. The ICD-10 classification of mental and behavioural disorders: diagnostic criteria for research. Geneva: World Health Organization 1993.

20. Oldfield RC. The assessment and analysis of handedness: the Edinburgh inventory. Neuropsychologia 1971; 9:97-113.

21. Uetsuki M, Matsuoka K, Kim Y, Araki T, Suga M et al. Estimation of premorbid IQ by JART in schizophrenia. Seishinigaku 2006; 48:15-22 [in Japanese].

22. Kane JM, Leucht S, Carpenter D, and Docherty JP. The expert consensus guideline series. Optimizing pharmacologic treatment of psychotic disorders. J Clin Psychiatry 2003; 64:1-100.

23. Key SR, Opler LA, Fiszbein A. (translated by Yamada H, Matsui K, Kikumoto K) Positive and Negative Syndrome Scale (PANSS) Rating Manual. Seiwa Shoten Publishers: 1991 [in Japanese].

24. Fukuda M. Near-infrared spectroscopy in psychiatry. Equilibrium Res 2010; 69:1-15 [in Japanese].

25. Okada E, and Delpy DT. Near-infrared light propagation in an adult head model, I. Modeling of low-level scattering in the cerebrospinal fluid layer. Appl Opt 2003; 42:2906-2914.

26. Okada E, and Delpy DT. Near-infrared light propagation in an adult head model, II. Effect of superficial tissue thickness on the sensitivity of the near-infrared spectroscopy signal. Appl Opt 2003; 42:2915-2922.

27. Baddeley A. The episodic buffer: a new component of working memory? Trends Cogn. Sci 2000; 4:417-423.

28. Jonides J, Smith EE, Koppe RA, Awh E, Minoshima S et al. Spatial working memory in humans as revealed by PET. Nature 1993; 363:623-625.

29. Leung HC, Gore JC, and Goldman-Rakic PS. Sustained mnemonic response in the human middle frontal gyrus during on-line storage of spatial memoranda. J Cogn Neurosci 2002; 14(4):659-671.

30. Strangman G, Culver JP, Thompson JH, and Boas DA. A quantitative comparison of simultaneous BOLD fMRI and NIRS recordings during functional brain activation. Neuroimage 2002; 17:719-731.

31. Paulesu E, Firth CD, and Frackowiak RS. The neural correlates of the verbal component of working memory. Nature 1993; 362:342-345.

32. Smith EE, Jonides J, and Koppe RA. Dissociating verbal and spatial working memory using PET. Cerebral Cortex 1996; 6:11-20.

33. D'Esposito M, Detre JA, Alsop DC, Shin RK, Atlas S et al. The neural basis of the central executive system of working memory. Nature 1995; 378:279-281.

34. Glahn DC, Therman S, Manninen M, Huttunen M, Kaprio $\mathrm{J}$ et al. Spatial working memory as an endophenotype for schizophrenia. Biol Psychiatry (Society of Biological Psychiatry) 2003; 53:624-626.

35. Park S, Holzman PS, and Goldman-Rakic PS. Spatial working memory deficits in the relatives of schizophrenic patients. General Psychiatry 1995; 52:821-828.

36. McGurk SR, Carter C, Goldman R, Green MF, Marder SR et al. The effects of clozapine and risperidone on spatial working memory in schizophrenia. Am J Psychiatry 2005; 162:1013-1016.

37. Reilly JL, Harris MS, Keshavan MS, and Sweeney JA. Adverse effects of risperidone on spatial working memory in first-episode schizophrenia. General Psychiatry 2006; 63:1189-1197.

38. Worsley MM, and Park S. Spatial working memory deficits in schizophrenia patients and their first degree relatives from Palau, Micronesia. Am J Med Genet 2002; 114:609-615.

39. Zabala A, Rapado M, Arango C, Robles O, de la Serna E et al. (2010) Neuropsychological functioning in early-onset first-episode psychosis: comparison of diagnostic subgroups. Eur Arch Psychiatry Clin Neurosci 2010; 260:225-233. 